\title{
Effects of Different Laser Treatments on Some Properties of the Zirconia-Porcelain Interface
}

\author{
Abdulsatar M Abdulsatar', Basima M A Hussein ${ }^{1 *(\mathbb{D})}$, Ali M Mahmood ${ }^{1}$ \\ ${ }^{1}$ Institute of Laser for Postgraduate Studies, University of Baghdad, Baghdad, Iraq
}

\section{*Correspondence to} Basima Mohammed Ali Hussein, Tel: 007947901491072 Email: basma.moh@ilps uobaghdad.edu.iq

Published online February 13 2021

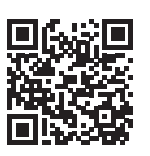

\section{Introduction}

Zirconia is a highly attractive dental material compared to other dental ceramics because of its mechanical properties, such as high flexural strength (700 to 1200 $\mathrm{MPa}$ ) and fracture toughness (7 to $10 \mathrm{MPa} \mathrm{m} 1 / 2$ ), and its optical properties. ${ }^{1}$

It is the material chosen by dentists for many dental applications and has been used as a basic material for single crowns, frameworks for fixed partial dentures, orthodontic brackets, endodontic post and implant abutments. $^{2}$

Zirconia has three phases: (1) Monoclinic (m) below $1170^{\circ} \mathrm{C}$, (2) Tetragonal (t) above $1170^{\circ} \mathrm{C}$, and (3) Cubic (c) above $2370^{\circ} \mathrm{C}$. After firing, the tetragonal-monoclinical phase transformation takes place during cooling, and the material changes from a tetragonal structure to a monoclinic, resulting in a volume increase of $3 \%$ to $5 \%$. Some fractures and cracks can form because of the compressive-stresses in the lattice. ${ }^{3}$

Zirconia is a material with a strengthened frame, and it must be sheltered with a semi-transparent veneering porcelain to get an esthetic appearance. ${ }^{4}$ Effective bonding relies on micro-mechanical interlocking between zirconia and the veneering porcelain, which is a very essential factor for the longevity of zirconia restorations. ${ }^{5}$ Unlike zirconia, veneering porcelain does not have good mechanical properties, and the nonexistence of strong bonding at the interface between zirconia and the veneering porcelain may result in fractures in the dental restoration. ${ }^{6}$

Factors affecting bond strength, including mechanical and chemical bonding, residual stress, and wettability, can affect the bonding between zirconia and porcelain. ${ }^{7,8}$

Therefore, to increase the bond strengths of veneering porcelain, some surface treatment procedures on zirconia have been developed such as: the application of the liners, mechanical surface roughening, salinization, tribiochemistry, thermal spray, chloro-silane treatment with steam, fusion with glassy balls, selective infiltration etching which creates intergranular porosity, corrosion with hot solutions, complex primer that reacts with hydroxyl groups and laser treatments. ${ }^{9}$

Previous studies have employed different lasers such as CO2, Nd;YAG and Er;YAG for the surface treatment of zirconia and reported varying degrees of success. ${ }^{10}$ Soltaninejad et al concluded that the surface treatment 
with Nd:YAG laser resulted in increased share bond strength and surface roughness. ${ }^{11}$ while Liu et al found that Nd:YAG laser cannot improve the surface roughness of zirconia. ${ }^{12}$ Moser et al used $\mathrm{CO}_{2}$ laser and concluded that laser treatment of zirconia increased the shear bond strength (SBS) values significantly. ${ }^{13}$

The aim of this study was to evaluate the effects of various laser surface-treatment (fractional CO2 laser and Q-switched Nd:YAG laser on pre- sintered zircon) on the bond strength between the veneering ceramics and zirconia

\section{Materials and Methods \\ Fabrication of Zirconia Specimens}

Fifty-nine discs ( $9 \mathrm{~mm}$ diameter ${ }^{*} 4 \mathrm{~mm}$ thickness) were prepared using the Y-TZP zirconia block (inCoris TZI C, Sirona, Germany). First of all, the cylinder model was designed by $\mathrm{Pc}$, and then the data were exported to the CAD/CAM system (inLab CAD/CAM system, Sirona, Germany) for milling the zirconia block and producing the specimens. The bonding surface of zirconia specimens was polished using $(800,1500,2000)$ grit silicon carbide abrasive paper for 15 seconds to standardized the surface roughness. ${ }^{14}$ After that, all specimens were cleaned ultrasonically using an ultrasonic cleaner with distilled water for 10 minutes to remove any contaminants. The samples were divided to form three groups:

\section{First Group (Control Group)}

Eleven pre-sintered zirconia specimens were left without laser surface treatment and sintered in a furnace (inFire HTC speed, Sirona, Germany) according to manufacturer's instructions, considered as a control group. The sample was tested by the light microscope for the assessment of surface morphology followed by the atomic force microscope (AFM) test. Ten samples were prepared for porcelain buildup according to the manufacturer instructions. These specimens were designed to test SBS testing. Each sample was attached to a piece of sticky wax ( $5 \mathrm{~mm}$ diameter ${ }^{\star} 3 \mathrm{~mm}$ thickness). After that, the samples were placed in a rubber mold and the investment material was poured into the mold. Then wax elimination was done using a hot steamer. Dental porcelain (VITA VM9, VITA Zahnfabirk, Germany) was applied to fill the mold after wax elimination according to the manufacturer's instructions and placed in the porcelain furnace (Programat P500, Ivoclar Vivadent, Germany).

After porcelain sintering, the investment material was removed and porcelain fused to zirconia samples was produced. All samples were soaked into a water bath filled with distilled water at $37^{\circ} \mathrm{C}$ for 24 hours before the SBS test. ${ }^{15}$ Each sample was attached to the universal testing machine (Instron 1195, England) and the load was applied at $1 \mathrm{~mm} / \mathrm{min} .{ }^{14}$ with the blade tip perpendicular to the zirconia porcelain interface. SBS was calculated using the formula below ${ }^{15}$ :

Shear bond strength $(\mathrm{MPa})=$ applied force $(\mathrm{n}) /$ area $\left(\mathrm{mm}^{2}\right)$

$\mathrm{SBS}=\mathrm{n} /$ area

\section{Second Group (CO2 Group)}

Thirty two pre sintered zirconia specimen surfaces were irradiated with the fractional $\mathrm{CO}_{2}$ laser10600 nm $\left(\mathrm{CO}_{2}\right.$ Fractional laser Brouchure, JHC1180, China). To estimate the most effective laser parameters, a pilot study was conducted using a laser beam with different pulse durations $(0.5,1,2,3,4,5,10$ milliseconds) and different output powers $(10,20,30 \mathrm{~W})$ while the time interval at 0.1 $\mathrm{ms}$, distance between spots $0.3 \mathrm{~mm}$ and number of scan 4 were fixed. After laser surface treatment, the specimens were sintered in a furnace (inFire HTC speed, Sirona, Germany) according to the manufacturer's manufacture instructions, following sintering, all samples were examined by the light microscope. To test SBS between laser treated zirconia and porcelain; a porcelain buildup and sintering was performed in the same procedure applied in the control group. Results of the pilot study including SBS test and surface morphology examination indicated that $30 \mathrm{~W}, 2 \mathrm{~ms}, 1 \mathrm{~ms}$, distance between spots $0.3 \mathrm{~mm}$ and number of scan 4 are the best parameters showed high SBS with no microcracks. Ten samples were prepared as $\mathrm{CO}_{2}$ group treated with these laser parameters and subjected to the SBS test after porcelain build-up. The AFM test was also performed for this group to assess the surface roughness.

\section{Third Group (Nd:YAG Group)}

Sixteen pre-sintered zirconia specimen surfaces were irradiated with the Q-SWITCHED Nd:YAG laser with a 1064-nm wavelength. The laser beam was delivered with an energy density of $15,20,25,30,35 \mathrm{~J} / \mathrm{cm}^{2}$, while the frequency was fixed to $10 \mathrm{~Hz}$. Then the samples were sintered and tested by the light microscope and SBS test. Results of the pilot study indicated that $30 \mathrm{~J} / \mathrm{cm}^{2}$ and 10 $\mathrm{Hz}$ are the best parameters gave high SBS with no micro cracks in the tested samples. Ten samples were prepared as a Nd:YAG group and treated with these laser parameters. Porcelain build-up was performed in the same manner described in the control group and subjected to the SBS test. For this group, the AFM test was also performed.

\section{Fracture Mode}

After the SBS test, the deboned surface of the zirconia was examined microscopically with $(\times 40)$ to determine the mode of fracture 15 as follows:

1. Adhesive failure: If less than $25 \%$ of the zirconia cylinder surface was covered with porcelain.

2. Cohesive failure: If less than $25 \%$ of the zirconia cylinder surface was visible.

3. Mixed failure: for all other cases. 


\section{Statistical Analysis}

Descriptive statistics were computed for SBS. Statistical Methods were used in order to analyze and assess the data, including descriptive statistics (mean and standard deviation) and inferential statistics. The ANOVA test was carried out to examine the significant difference between the tested groups $(P<0.05)$. The Tukey HSD test was carried out to detect the significant differences between the two groups.

\section{Results}

\section{Light Microscopic Observations}

The photographs below show the surface morphology of the zirconia specimens (Figures 1A, B, C). The image of the untreated zirconia appears to be smoother than that of $\mathrm{Nd}: \mathrm{YAG}$ and $\mathrm{CO}_{2}$, and the surface texture of the laser-treated zirconia consists of micro-retentive pits with different degrees of roughness between the two lasers. There is no microcrack or defects appear in the microscopy images.

\section{Shear Bond Strength}

The mean SBS and standard deviations are shown in Table 1. In general, the laser surface treatment increased the SBS of zirconia to porcelain. The highest bond strength was obtained from the $\mathrm{CO}_{2}$ group (23.6 MPa). The lowest value was obtained from the control group (7.397 MPa). The bond strength of the Nd:YAG group was lower than that of the $\mathrm{CO}_{2}$ group (20.180 MPa).

To test the significant difference between groups, ANOVA was used. Table 2 shows a highly significant difference between the control and the experimental groups.

Table 3 shows that there is a significant difference between the control and the experimental groups, but there is a non-significant difference between the two laser treated groups.

\section{Mode of Failure}

Failure types are shown in Table 4 . The analysis of the mode of failure after the SBS test revealed that the adhesive failure was the predominant mode in the control group, but the cohesive failure had the highest frequency

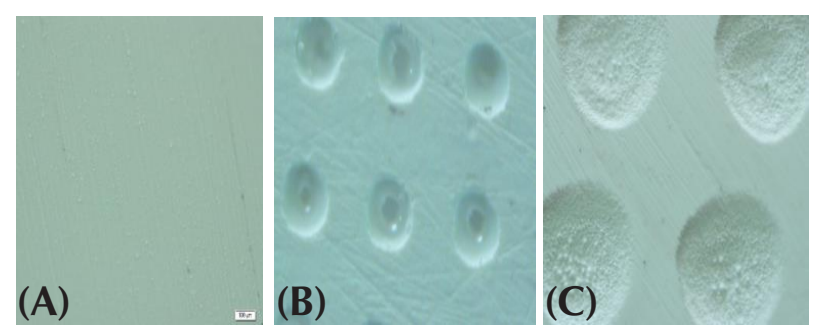

Figure 1. Light Microscopy Pictures With (×100), A. Control, B. Fractional $\mathrm{CO}_{2^{\prime}}$ C. Q switched Nd:YAG.
Table 1. Mean and Standard Deviation (SD) of the SBS in the Tested Groups

\begin{tabular}{lcc}
\hline Group & Mean SBS (MPa) & SD \\
\hline Control & 7.397 & 2.288 \\
Fractional $\mathrm{CO}_{2}$ laser & 23.603 & 4.007 \\
Nd:YAG laser & 20.180 & 5.339 \\
\hline
\end{tabular}

Table 2. Shear Bond Strength ANOVA Test Between Groups

\begin{tabular}{lccccc}
\hline & Sum of Squares & df & Mean Square & F & P Value \\
\hline Between groups & 1459.188 & 2 & 729.594 & 43.959 & 0.00 \\
Within groups & 448.127 & 27 & 16.597 & & \\
Total & 1907.315 & 29 & & & \\
\hline
\end{tabular}

Table 3. Tukey Test Between Control and Experimental Groups

\begin{tabular}{lcccc}
\hline I Group & J Group & Mean Difference (I-J) & Std. Error & $\boldsymbol{P}$ Value \\
\hline \multirow{2}{*}{ Control } & $\mathrm{CO}_{2}$ & -16.20600 & 1.82194 & 0.000 \\
& $\mathrm{Nd}$ :YAG & -12.78300 & 1.82194 & 0.000 \\
\multirow{2}{*}{$\mathrm{CO}_{2}$} & Contro; & 16.20600 & 1.82194 & 0.000 \\
& $\mathrm{Nd}$ :YAG & 3.42300 & 1.82194 & 0.164 \\
\multirow{2}{*}{ Nd:YAG } & Control & 12.78300 & 1.82194 & 0.000 \\
& $\mathrm{CO}_{2}$ & -3.42300 & 1.82194 & 0.164 \\
\hline
\end{tabular}

Table 4. Mode of Failure Results of the Tested Groups

\begin{tabular}{lccc}
\hline Mode of Failure & Control & $\mathbf{C O}_{2}$ & Nd;YAG \\
\hline Adhesive & 6 & 1 & 2 \\
cohesive & 0 & 8 & 3 \\
mixed & 4 & 2 & 5 \\
\hline
\end{tabular}

in the $\mathrm{CO}_{2}$ groups while in the Nd:YAG group the mixed mode of failure was the highest mode

\section{Surface Roughness Analysis}

Surface roughness was evaluated by AFM. Table 5 displays surface roughness measurements of the different groups. The lowest Ra value was observed in the control specimen (12.9) followed by that

Nd;YAG laser treated group (21.6) and the highest Ra value was recorded in the with $\mathrm{CO}_{2}$ group (28.6).

\section{Discussion}

Some studies have evaluated the bond strength between the zirconia and veneering porcelain and the surface roughness after various surface treatments on zirconia. ${ }^{17,18}$ Effective bonding is essential to ensure the longevity of the zirconia dental restoration; otherwise, there will be deboning of the porcelain. To remove this failure, researchers aim to improve the bond strength by increasing the surface area of zirconia through different surface-treatments. ${ }^{19}$

Moon et al applied surface treatments to pre-sintered zirconia. It was highlighted that pre -sintered surface 
Table 5. Surface Roughness Measurements by the AFM Test of the Tested Group

\begin{tabular}{lcc}
\hline Control & $\mathrm{CO}_{2}$ & Nd:YAG \\
\hline 12.9 & 27.6 & 21.6 \\
\hline
\end{tabular}

treatment had many benefits. It was concluded that in post-sintered zirconia laser surface treatment, microcracks were not occurring. ${ }^{20}$ It has been reported that postsintering surface treatments increase the fracture risk by weakening the structure of zirconia and accelerating the tetragonal to monoclinical transformation. ${ }^{21}$

The laser has been suggested by many authors as an alternative surface treatment method to for increasing the surface roughness of zirconia. ${ }^{22-24}$ Therefore, the focus of the present study was to investigate the effect of fractional $\mathrm{CO}_{2}$ and Q switched Nd:YAG laser irradiation (at different intensities) on the bond strength of Y-TZP to porcelain prior to sintering.

Shear stresses are thought to be major stresses leading to bonding-failure of restorative materials, and the SBS tests are simple, quick and appropriate. ${ }^{11}$ In this study, the SBS test was used to examine the bonding of zirconia ceramics to porcelain.

The result of this study showed that the use of the fractional $\mathrm{CO}_{2}$ laser or the Q switched Nd:YAG laser on the surface of zirconia resulted in significantly improved SBSs compared to the control group, because of the increase of surface roughness on the zirconia surface that enhanced the interlocking with the porcelain. The effect of the laser could be related to the vaporization or ablation of the zirconia material through the photothermal process. The vaporization is considered as a micro- explosion of portions of material heated above the melting point. ${ }^{25}$

This result is in agreement with that of a study by Ahrari et al who concluded that laser surface treatment on zirconia significantly increased SBS because of surface roughness. ${ }^{10}$ However, the result disagrees with Kirmali et al; they proposed that $\mathrm{Nd}$; YAG lasers are not effective to in increasing the bond strength between zirconia and porcelain. This could be due to the fact that the authors in the study used different laser energy and different pulse duration. ${ }^{14}$ Alhussani and Jawad concluded that irradiation of zirconia with the fractional $\mathrm{CO}_{2}$ laser could be mentioned as a suitable alternative methods of zirconia conventional surface treatment. ${ }^{26}$

The morphology analysis in the present study showed an increase in the surface area of zirconia without any microcracks after laser irradiation. This finding disagrees with other studies. Akyıl et al and Gorler and Ozdemir reported cracks on the zirconia surface after laser treatment. This disagreement may be related to laser irradiation on fully- sintered zirconia. ${ }^{15,27}$

In the study, it was observed that the number of adhesive failure types was less than that for mixed and cohesive failure types in laser treated groups because adhesive failure is related to low bond strength. ${ }^{28}$ This finding is in agreement with Cevik and colleagues study in which the adhesive mode of failure is less in the laser treated group than the control group. ${ }^{17}$ Cavalcanti et al examined the bond types after the surface treatments between $\mathrm{ZrO}_{2}$ and resin cement and found that the adhesive failure type was higher than the mixed failure type in all groups. ${ }^{29,30}$ The reason may have been because surface treatments on $\mathrm{ZrO}_{2}$ were applied after sintering

\section{Conclusion}

Within the limitations of this study, the following conclusions are drawn:

1. Both Fractional $\mathrm{CO}_{2}$ laser and $\mathrm{Q}$ switched Nd:YAG laser treatments demonstrated higher bond strength than untreated zirconia and there is no significant difference in SBS between the experimental groups.

2. Laser surface treatment can change the surface roughness, surface morphology of the pre sintered zirconia and the fracture mode between zirconia and porcelain.

3. Laser parameters are a critical factor that affects the surface treatment final result.

\section{Acknowledgment}

Authors appreciate the cooperation of all members in the Institute of Laser for Postgraduate Studies, University of Baghdad.

\section{References}

1. Vagkopoulou T, Koutayas SO, Koidis P, Strub JR. Zirconia in dentistry: Part 1. Discovering the nature of an upcoming bioceramic. Eur J Esthet Dent. 2009;4(2):130-51

2. Madfa AA, Al-Sanabani FA, Al-Qudami NH, AlSanabani JS, Amran AG. Use of zirconia in dentistry: An overview. Open Biomater J. 2014;5(1):1-79. doi: 10.2174/1876502501405010001

3. Piconi C, Maccauro G. Zirconia as a ceramic biomaterial. Biomaterials. 1999; 20(1):1-25. doi: 10.1016/s01429612(98)00010-6

4. Sundh A, Molin M, Sjögren G. Fracture resistance of yttrium oxide partially-stabilized zirconia all-ceramic bridges after veneering and mechanical fatigue testing. Dent Mater. 2005;21(5):476-482. doi: 10.1016/j.dental.2004.07.013

5. Omidi BR, Yeganeh PK, Oveisi S, Farahmandpour N, Nouri F. Comparison of micro-shear bond strength of resin cement to zirconia with different surface treatments using universal adhesive and zirconia primer. J Lasers Med Sci 2018; 9(3):200-206 doi: 10.15171/jlms.2018.36.

6. Bona A D, Pecho OE, Alessandretti R. Zirconia as a dental biomaterial. Materials (Basel). 2015;8(8):4978-4991. doi: 10.3390/ma8084978.

7. Isgrò G, Pallav P, van der Zel JM, Feilzer AJ. The influence of the veneering porcelain and different surface treatments on the biaxial flexural strength of a heat-pressed ceramic. J Prosthet Dent. 2003;90(5):465-473. doi: 10.1016/j. prosdent.2003.08.003.

8. De Jager N, Pallav P, Feilzer AJ. The influence of design 
parameters on the FEA-determined stress distribution in CAD-CAM produced all-ceramic dental crowns. Dent Mater. 2005;21(3):242-251. doi: 10.1016/j. dental.2004.03.013.

9. Sato H, Yamada K, Pezzotti G, Nawa M, Ban S. Mechanical properties of dental zirconia ceramics changed with sandblasting and heat treatment. Dent Mater J. 2008; 27(3):408-414. doi: 10.4012/dmj.27.408.

10. Ahrari F, Boruziniat A, Mohammadipour HS, Alirezaei M. The effect of surface treatment with a fractional carbon dioxide laser on shear bond strength of resin cement to a lithium disilicate-based ceramic. Dent Res J (Isfahan). 2017;14(3):195-202. doi: 10.4103/1735-3327.208762.

11. Soltaninejad F, Moezizadeh M, Khatiri M, Razaghi H, Namdari M, Nojehdehian $\mathrm{H}$. Effect of Nd: YAG laser energy density on bond strength and phase transformation of zirconia. Biomed Res (Aligarh). 2017;28(17):7341-7347.

12. Liu L, Liu S, Song X, Zhu Q, Zhang W. Effect of Nd: YAG laser irradiation on surface properties and bond strength of zirconia ceramics. Lasers Med Sci. 2015;30(2):627-634. doi: 10.1007/s10103-013-1381-7.

13. Moser D, Fish S, Beaman J, Murthy J. Multi-layer computational modeling of selective laser sintering processes. Proceedings of the ASME 2014 international mechanical engineering congress and exposition; $2014 \mathrm{Nov}$ 14-20; Montreal, Quebec, Canada. New York: American Society of Mechanical Engineers; 2015. doi: 10.1115/ IMECE2014-37535.8.

14. Kirmali O, Akin H, Ozdemir AK. Shear bond strength of veneering ceramic to zirconia core after different surface treatments. Photomed Laser Surg. 2013; 31(6):261-8. doi:10.1089/pho.2013.3487

15. Gorler O, Ozdemir AK. Bonding strength of ceromer with direct laser sintered, Ni-Cr-based, and $\mathrm{ZrO}_{2}$ metal infrastructures after Er:YAG, Nd:YAG, and Ho:YAG laser surface treatments-A comparative in vitro study. Photomed Laser Surg. 2016;34(8):355-62. doi:10.1089/ pho.2016.4129.

16. Tsuo Y, Yoshida K, Atsuta M. Effects of alumina-blasting and adhesive primers on bonding between resin luting agent and zirconia ceramics. Dent Mater J. 2006;25(4):66974. doi: 10.4012/dmj.25.669.

17. Cevik P, Cengiz D, Malkoc MA. Bond strength of veneering porcelain to zirconia after different surface treatments. J Adhes Sci Technol. 2016;30(22):2466-2476. doi: 10.1080/01694243.2016.1184779.

18. Kirmali O, Kapdan A, Kustarci A, Er K. Veneer ceramic to Y-TZP bonding: comparison of different surface treatments. J Prosthodont. 2016;25(4):324-9. doi: 10.1111/ jopr.12304.

19. Raigrodski AJ, Chiche GJ, Potiket N, Hochstedler JL, Mohamed SE, Billiot S, et al. The efficacy of posterior three-unit zirconium-oxide-based ceramic fixed partial dental prostheses: A prospective clinical pilot study.
J Prosthet Dent. 2006;96(4):237-44. doi: 10.1016/j. prosdent.2006.08.010.

20. Moon JE, Kim SH, Lee JB, Ha SR, Choi YS. The effect of preparation order on the crystal structure of yttriastabilized tetragonal zirconia polycrystal and the shear bond strength of dental resin cements. Dent Mater. 2011;27(7):651-63. doi: 10.1016/j.dental.2011.03.005.

21. Guess PC, Zhang Y, Kim J-W, Rekow ED, Thompson VP. Damage and reliability of Y-TZP after cementation surface treatment. J Dent Res. 2010;89(6):592-6. doi: $10.1177 / 0022034510363253$.

22. Li R, Ren Y, Han J. [Effects of pulsed Nd: YAG laser irradiation on shear bond strength of composite resin bonded to porcelain]. Hua Xi Kou Qiang Yi Xue Za Zhi. West China J Stomatol. 2000;18(6):377-379. [Chinese].

23. Da Silveira BL, Paglia A, Burnett Jr LH, Arai Shinkai RS, Eduardo CDP, Spohr AM. Micro-tensile bond strength between a resin cement and an aluminous ceramic treated with Nd: YAG laser, Rocatec System, or aluminum oxide sandblasting. Photomed Laser Surg. 2005;23(6):543-8. doi: 10.1089/pho.2005.23.543.

24. Spohr AM, Borges GA, Júnior LHB, Mota EG, Oshima HMS. Surface modification of In-Ceram zZirconia ceramic by Nd: YAG laser, Rocatec system, or aluminum oxide sandblasting and its bond strength to a resin cement. Photomed Laser Surg. 2008;26(3):203-8. doi: 10.1089/ pho.2007.2130.

25. Meister J, Franzen R, Forner K, Grebe H, Stanzel S, Lampert $\mathrm{F}$, et al. Influence of the water content in dental enamel and dentin on ablation with erbium YAG and erbium YSGG lasers. J Biomed Opt. 2006;11(3):34030. doi: 10.1117/1.2204028.

26. Alhassani LI, Jawad HA. Influence of Fractional CO2 Laser Irradiation on Temperature Elevation and Bonding Strength of Resin Cement to the Zirconia Ceramic. Iraqi $J$ Laser. 2018;17:23-31.

27. Akyıl MŞ, Uzun İH, Bayındır F. Bond strength of resin cement to yttrium-stabilized tetragonal zirconia ceramic treated with air abrasion, silica coating, and laser Irradiation. Photomed Laser Surg. 2010;28(6):801-8. doi: 10.1089/pho.2009.2697.

28. Toledano M, Osorio R, Osorio E, Aguilera FS, Yamauti M, Pashley DH, et al. Durability of resin-dentin bonds: effects of direct/indirect exposure and storage media. Dent Mater. 2007;23(7):885-92. doi: 10.1016/j.dental.2006.06.030.

29. Cavalcanti AN, Pilecki P, Foxton RM, Watson TF, Oliveira MT, Gianinni M, et al. Evaluation of the surface roughness and morphologic features of Y-TZP ceramics after different surface treatments. Photomed Laser Surg. 2009;27(3):473-9. doi: 10.1089/pho.2008.2293.

30. Reem Mounes Dawood and Adel F. Ibraheem. Evaluation of shear bond strength of zirconia to tooth structure after different zirconia surface treatment techniques. J Bagh College Dentistry. 2015, 27(1):77-85 